\title{
Primo Vascular Node in the Bone Marrow and Longevity
}

\author{
Vitaly Vodyanoy ${ }^{1,2, *}$, Oleg Pustovyy ${ }^{1}$, Ludmila Globa ${ }^{1}$ \\ ${ }^{1}$ Department Anatomy, Physiology and Pharmacology, College of Veterinary Medicine, Auburn University, Auburn, AL, USA \\ ${ }^{2}$ School of Kinesiology, Auburn University, Auburn, AL, USA
}

Received June 21, 2021

Revised October 27, 2021

Accepted December 6, 2021

Correspondence to

Vitaly Vodyanoy

Department Anatomy, Physiology and

Pharmacology, College of Veterinary

Medicine, Auburn University, Auburn,

AL, USA

E-mail vodyavi@auburn.edu
Background: Intra-organic bone marrow node is predicted to be a part of the primo vascular system that plays a critical role in hematopoiesis and generation and regeneration of other cells. Two models of cell regeneration were suggested, one involving DNA synthesis and the other pertaining to DNA recycling.

Objectives: The aim of this work is to extract a primo node from bone marrow, characterize its structure, understand its biochemistry and cell composition, and suggest a cell regeneration mechanism.

Methods: Primo nodes were sampled from segmented halves of the rat femur. We used immunohistochemistry and high-resolution fluorescent microscopy to analyze 1200 samples obtained from 42 rats and 190 primo nodes.

Results: Primo nodes in the bone marrow have an oval or round structure of about one millimeter in diameter, which is encompassed by a fine capsule, having incoming and outgoing vessels filled with the extracellular matrix and hematopoietic, mesenchymal, endothelial stem cells, as well as cells of the megakaryocyte family found in other primo nodes.

Conclusion: Our findings imply that bone marrow nodes are intra-organic primo vascular nodes, and they provide ways and approaches for further investigation. Bone marrow nodes are simple to examine ex vivo in a variety of environments to assess cell regeneration mechanisms, wound healing, and organism rejuvenation and lifespan. Further research into these and other intra-organic nodes in animals and humans could lead to new regenerative medicine and longevity strategies that have yet to be discovered.

Keywords: Primo vascular system, Regeneration, Acupuncture, Microcell, Stem cell, Microscopy

\section{INTRODUCTION}

All cells in the human body have a relatively short lifetime. The lifetime of some of the major building blocks of cells, such as proteins, is even shorter. Many cells and their constituents are replaced every day. This means that when we wake up every morning, we are practically newborns. If the biochemical mechanisms of rejuvenation work perfectly and we do not meet with a fatal accident, we should ideally live forever. However, we do not. Why? The main reason is in the mechanisms of cell death and DNA replication.

According to contemporary science, the process of programmed cell death, apoptosis, leads to morphological cell changes and death. Some of these changes are blebbing, cell shrinkage, and nuclear fragmentation [1]. During the fragmentation, chromosomes are packed in so-called apoptotic bodies that are expelled outside the dying cell. The phagocytic cells engulf and digest the apoptotic bodies and destroy the chromosomes. To sustain life, the dead cells need to be replaced. This cell replenishment is assumed to occur through the replication of stem cells. The stem cells divide, and a daughter cell replaces a dead cell. Every time a stem cell divides and its DNA replicates, it has to copy and transmit the same sequence of 3.7 billion nucleotides [2] to its daughter cells. While most DNA replicates with reasonably high accuracy, mistakes (mutations) do happen [3]. The reported mutation rates at the time of replicating human DNA is one mistake per 100 to 1000 nucleotides [4]. Since about 5070 billion cells die each day due to apoptosis in an average human adult and 6 billion bases are in the human genome, one can estimate that in the worst-case scenario, mistakes accumulated for 30 years can reach up to $20 \%$ of all 37 trillion cells of the human body (Supplementary Materials). Of course, not all mutations are harmful, and DNA repair fixes 
many mistakes. However, some of the mutations could cause cancer. Three are other detrimental consequences of the errors in cell duplications. One of them is telomere-dependent replicative cellular senescence [5]. Due to the nature of DNA replication, telomeres become progressively shorter with every round of human cell division [6]. One of the essential telomere functions is preventing chromosomal end-to-end fusions and genomic instability [5]. Importantly, telomere shortening is linked to obesity [7] and atherosclerosis [8] and is a serious contributor to aging. Cell renewal through stem cell duplication suffer also from stem cell aging [9]. Among other causes, stem cell aging decreased per-cell repopulating activity, self-renewal, and increased apoptosis with stress [10]. Vascular calcification [11] and glycosylation [12] also contribute to aging, but the main "evil" in our life is the DNA replication that causes mutations. It seems impossible to replenish naturally dying cells without cell division and DNA replication; however, it is indeed possible.

About 60 years ago, when stem cell science was in its infancy, Korean scientist Kim [13] discovered and studied a new primo vascular system (PVS) whose primary function is to regenerate cells and tissues. In 2002, a research team in Seoul National University reopened the PVS research, defined the existence of PVS in various organs, and discovered new characteristics of PVS [14]. The PVS is composed of very thin vessels(diameter of 20-50 $\mu \mathrm{m}$ ) and small nodes $(<1500 \mu \mathrm{m})$ that are connected to primo vessels. Dr. Kim reported that the replacement of dying cells could be achieved without cell division and DNA replication by the recycling of the DNA of the expiring cells [15]. According to Kim's model, the cell that is preparing to die experiences chromatin condensation and are packed into membrane-bound capsules; the deformation and rupture of the nuclear membranes result in the release of these capsules into the cytoplasm, and the rupture of the external membrane releases the capsules to the outside. Kim named these capsules sanals and claimed that each sanal contains a single chromosome. Therefore, if the dying human cell produces 46 sanals, then these sanals are subsequently used for cell regeneration. The process, described by Kim, is almost identical to apoptotic cell death and the generation of apoptotic bodies, but it has a major difference: phagocytic cells destroy apoptotic bodies. However, in contrast, PVS protects sanals and uses them to regenerate cells. The expulsion of the apoptotic bodies to the outside and their digestion by phagocytic cells mark the end of a cell's life, but the release of sanals that preserve DNA is the beginning of a new one. Kim further showed that the expelled cell sanals are collected by the PVS, assembled into the cell with a complete genome, and then positioned to replace a dead cell. The most significant factor in this process is that the DNA is recycled, and not duplicated or mutated. Kim demonstrated that both
DNA duplication and recycling mechanisms coexist for the replacement of dead cells. The DNA recycling method provides no errors and promises a longer life.

We can reproduce some of the experiments described by Kim [13]. We found conditions when some blood cells in vitro undergo the process of death with the release of sanals, small uniform $\sim 1-\mu \mathrm{m}$ size subcellular bodies, that tend to assemble in groups. In this study, rat primo vessels and nodes were harvested. From the nodes, we extracted and purified sanals, which, when viewed under a high-resolution microscope, looked like micron-size bubbles carrying a speck of matter inside. We evaluated sanals by high-resolution light microscopy [16]. We further identified vascularized node-like compartments in rat bone marrow that fit the requirements for stem cell niche, which we called hemmules [17]. In this aspect, we reported that bone marrow nodes, or hemmules, are the primo vascular nodes that belong to the PVS. The bone marrow primo vascular nodes manifested the structural and biochemical properties of the DNA recycling mechanism for cell regeneration that could save and prolong lives. We can reproduce some of the experiments described by Kim [13]. We found conditions when some blood cells in vitro undergo the process of death with the release of sanals, small uniform $\sim 1$ $\mu \mathrm{m}$ size subcellular bodies, that tend to assemble in groups. In this study, rat primo vessels and nodes were harvested. From the nodes, we extracted and purified sanals, which, when viewed under a high-resolution microscope, looked like micron-size bubbles carrying a speck of matter inside. We have demonstrated that sanals merge and create cell-like structures [18]. We further identified vascularized node-like compartments in rat bone marrow that fit the requirements for stem cell niche, which we called hemmules [17]. In this aspect, we reported that bone marrow nodes, or hemmules, are the primo vascular nodes that belong to the PVS. The bone marrow primo vascular nodes manifested the structural and biochemical properties of the DNA recycling mechanism for cell regeneration that could save and prolong lives.

The purpose of these studies is to extract the primo vascular nodes from rat femur bone. Using high-resolution fluorescent microscopy and immunochemistry, characterize the cellular composition of the nodes, the structure of the node vessel, and comparison with other primo nodes and vessels.

\section{MATERIALS AND METHODS}

\section{Animals}

Auburn University's Institutional Animal Care and Use Committee (AU IACUC) sanctioned the protocol of animals (protocol code 2014-2415). Adult male Sprague-Dawley rats (Envigo, Dublin, VA) weighing $\sim 300 \mathrm{~g}$ were considered 
for this study. The animals were maintained at a constant temperature of $25^{\circ} \mathrm{C}$, relative humidity $60 \%$, under a $12-\mathrm{h}$ light/dark cycle, and had access to food and water all the times.

\section{Microdissection and extraction of bone marrow nodes}

A detailed description of the procedure was presented in [17]. In short, we split a femur bone into two halves. The bone marrow nodes were dissected using surgical scissors and were fixed in Bouin's fluid (Electron Microscopy Sciences. PA, USA). Moreover, control samples of the bone marrow blood vessel, bone marrow, and lymph nodes were also collected. Representative samples, 190 primo nodes and 1200 sections, obtained from 42 rats were included in this work.

\section{Immunohistochemistry}

The sections were exposed to antibodies diluted in 5\% goat or donkey serum in smooth muscle's alpha actin (1:50, ThermoFisher Scientific; ab75769), PBS: Actin (1:100, Millipore; MAB1501), Fibronectin (1:50, ThermoFisher Scientific; 15613-1-AP), CD90 (1:100, ThermoFisher Scientific; MA1-80651), CD133 (1:20, ThermoFisher Scientific; 184701-AP), CD150 (1:50, ThermoFisher Scientific; ND600-408), LYVE-1 (1:100, ThermoFisher Scientific; ab9774), OCT4 (1:50, ThermoFisher Scientific; PA5-20887), NANOG (1:100, ThermoFisher Scientific; PA5-20889), REXO1 (1:20, ThermoFisher Scientific; ab16285), and vWF (1:20,
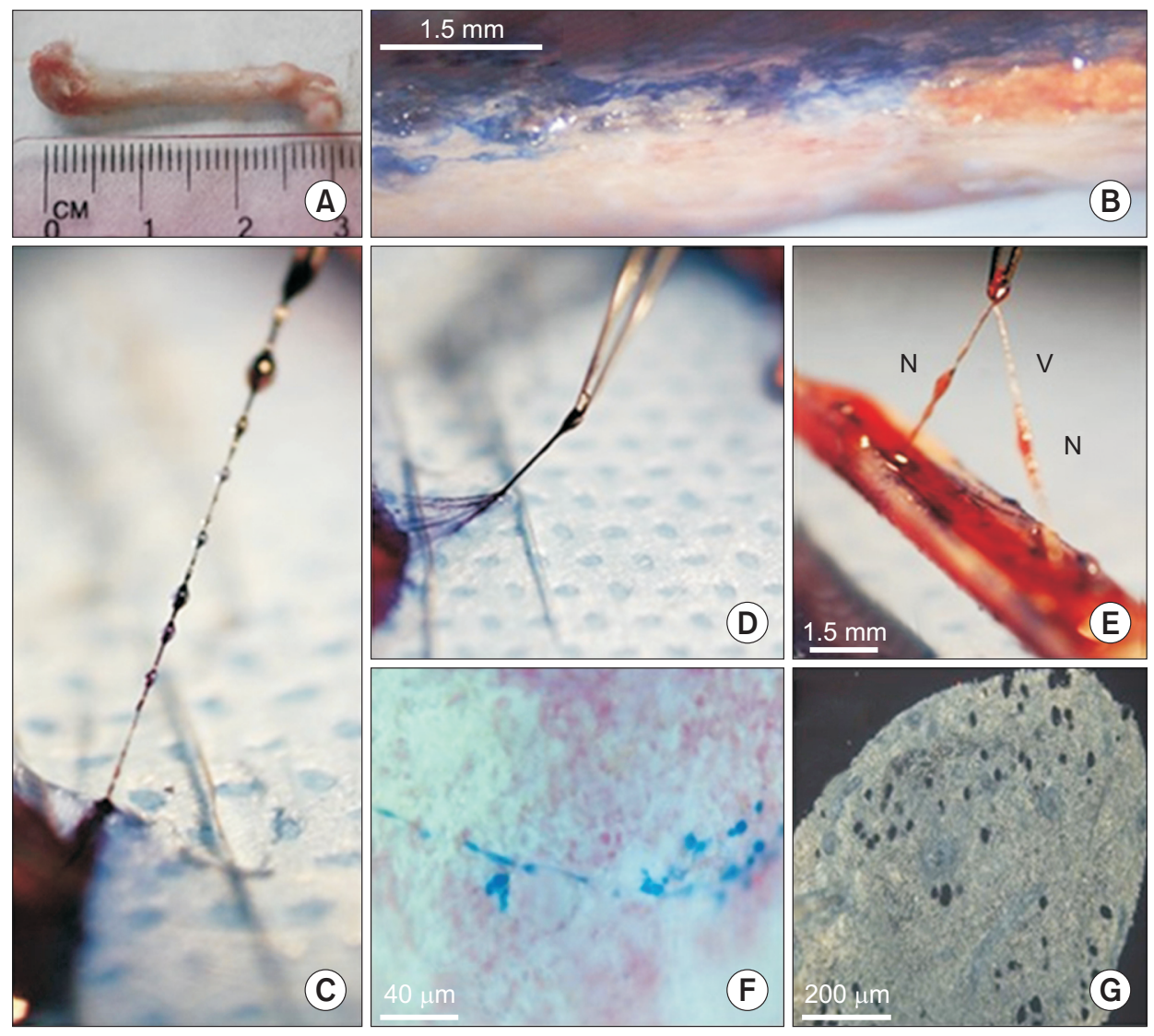

Fig. 1. The primo node and vessels taken from the freshly split rat femur bone. (A) Femur bone. (B) A split bone exposes a bone marrow stained by the trypan blue. (C) A series of primo nodes are connected to the vessel. The lower primo node is attached to the end primo node inside the bone marrow. The distance between the centers of the background dots is $1.5 \mathrm{~mm}$. (D) The efferent vessel of the lower end primo node is composed of multiple sub-vessels. (E) These primo nodes are extracted from the bone marrow, while both ends of the vessel are attached to the end primo nodes in the bone marrow. $\mathrm{N}=$ primo node, $\mathrm{V}=$ vessel. (F) A thin-branched vessel in the non-fixed bone marrow is stained by trypan blue. (G) A slice of the fixed primo node is extracted from the bone marrow. Openings. To characterize the structure of the primo nodes, we conducted high-resolution microscopy of the primo node thin sections. A typical longitudinal section of a primo node is illustrated in Fig. 2. The primo node of the oval shape of $600 \times 1600 \mu \mathrm{m}$ is surrounded by a fibrous capsule. In addition, the central vessel passing through the longitudinal direction almost through the entire primo node is visible on the surface of the slide, along with many smaller longitudinal vessels and channels. The longitudinal and transversal vessels create a single vascular system within the primo node. The extracellular matrix creates seemingly sponge-like tissues to provide small internal channels, which enables cells and other constituents to travel and interact within the primo node that are voids of large-sized cells [17]. 
ThermoFisher Scientific). Later, the slides were incubated with auxiliary antibodies, namely, Alexa Fluor 488 or Alexa Fluor 555 (1:500, ThermoFisher Scientific) dipped in blocking buffer ( $5 \%$ serum) in a dark zone at room temperature for almost an hour.

\section{High-resolution fluorescent microscopy}

The fluorescent optical system comprises of a speciallydesigned condenser [19] positioned in an Olympus BX51 microscope (Olympus America Inc., PA, USA) by replacing a bright-field condenser and allowing optical slicing at $90 \mathrm{~nm}$ resolution.

\section{RESULTS}

\section{Anatomy and fine structure of bone marrow primo node}

We removed primo nodes from the divided halves of femur rat bone, as described in the "Material and Methods" (Fig. 1).

Fig. 2 shows a representative longitudinal section of the primo node. A fibrous capsule encloses the primo node of an elongated shape of $600 \times 1600 \mu \mathrm{m}$. The central vessel having a diameter of about $50 \mu \mathrm{m}$ contains the lumen with thick walls girded by surrounding rib-like structures. The vessels create a single vascular system within the primo node. The extracellular matrix creates a spongiose tissue to support small internal channels, which allows node components to move and interact within the primo node. The bone marrow node is not very vascular compared to the organ surface node [20]. The extracellular matrix occupies the largest volume of the bone marrow node. The wall of the vessels is made up of several optically distinguished layers such as the outermost external Layer 1 (where cells are positioned along the vessel), the middle Layer 2 (transversal cells), the second middle layer, Layer 3 (where cells are positioned longitudinally), and the internal Layer 4 (longitudinal cells).

We used high magnification darkfield microscopy and optical slicing to reveal the vessel inside the node (Fig. 3). Fig. 3A presents the transversal section of the hemmule vessel. Panels (B-D) of Fig. 3 represent three consecutive longitudinal optical slices in close proximity to the top portion of the vessel (Fig. 3B), through the middle of the vessel (Fig. 3C), and in close proximity to the lower part of the vessel (Fig. 3D). Meanwhile the wall of the vessels comprises of several optically distinguished layers: the outmost external Layer 1 (cells are positioned along the vessel), the middle Layer 2 (transversal cells), the second middle layer (cells are positioned longitudinally), and the internal Layer 4 are composed of longitudinal cells. The characteristic features of Layer 2 are vining around the vessel thick fiber-like loops and longitudinal thin fiber treads that stretch parallel to the vessel in its entirety. This vessel is filled with small 3-4 $\mu \mathrm{m}$ cells.

Fig. 4 shows the interaction of the sub-vessel with the vascular endothelial cell marker anti-LYVE-1 antibody. The antibody did not stain cells of all four sub-vessel layers (including endothelial cells of Layer 4). Instead, the antibody
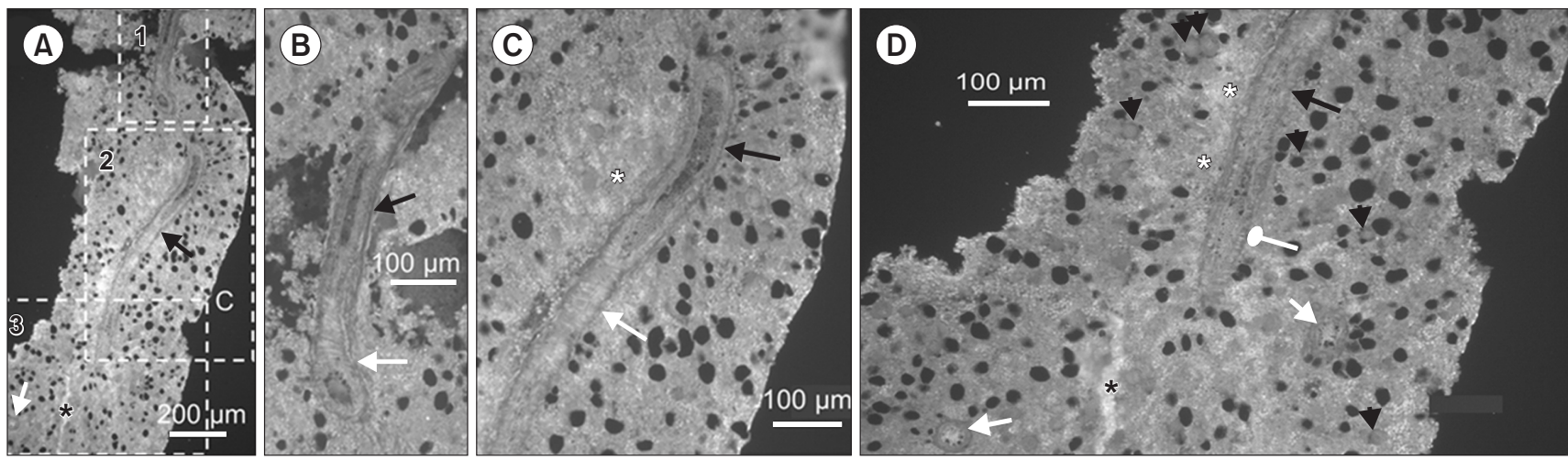

Fig. 2. Longitudinal section of the primo node. A section of $6 \mu \mathrm{m}$ from the rat femur bone marrow. (A) The section shows a portion of the central vessel passing in the longitudinal direction (black arrow). A smaller longitudinal vessel is labeled using an asterisk. The lumen of one transversal vessel is highlighted with a white arrow. Letter $\mathrm{C}$ depicts a fragment of the capsule. The section is divided based on three areas of interest and marked as 1, 2, and 3 and shown in panels (B-D), respectively. (B) The lower portion of the vessel (white arrow) illustrates the lumen with thick walls. A higher part of the vessel (black arrow) cuts open to expose a central duct with thick walls. (C) The magnified area 2 from panel (A). A white arrow reveals part of the vessel with a longitudinally ribbed wall surface. A black arrow points to the cut open vessel with thick walls. A white asterisk denotes an area with a high concentration of small vessels. (D) The central longitudinal vessel (long black arrow) is shown to be cut open (round head white arrow) before going down transversely into the slide. Two more large transverse vessels lumens are visible in this section (white arrows). A relatively large channel is labeled using a black asterisk. The entire area of the slice is covered with round holes, which shows the footprints of missing large size cells. Some of these cells are in close proximity to the holes (short black arrows). 

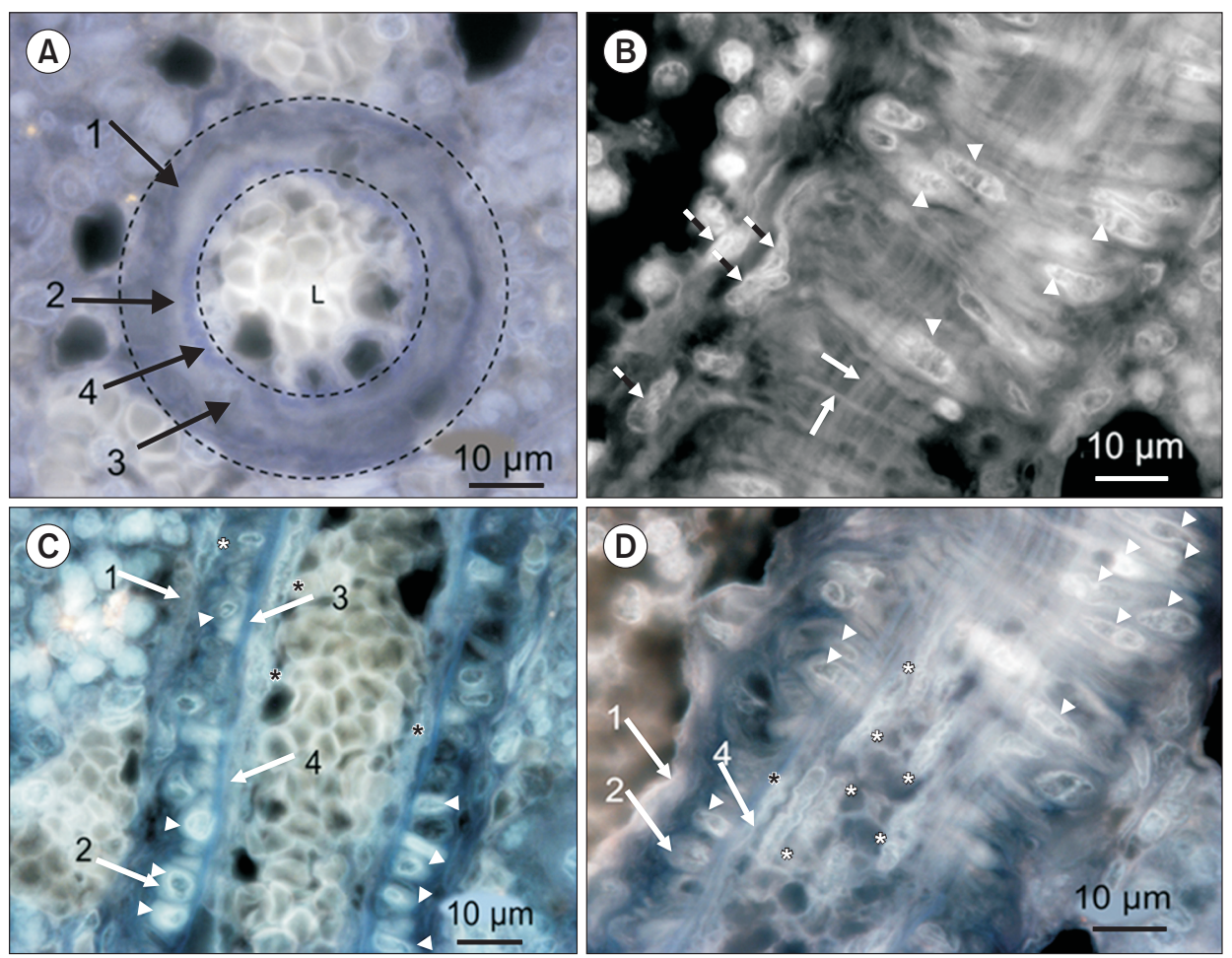

Fig. 3. High-magnification darkfield images of the vessel inside the primo node. (A) Transversal section of the primo node vessel. The wall of the vessels is composed of several optically distinguished Layers $1-4 ; \mathrm{L}=$ lumen. (B) The longitudinal section of the vessel: arrowheads = vascular smooth muscle cells comprised the thick fiber-like loops (Layer 2); solid arrows $=$ longitudinal, thin fiber treads, and transversal fiber; dotted arrows = longitudinal fibroblastic cells and their processes belonging to the vessel external Layer 1. (C) The longitudinal section cuts the middle portion of the vessel. White arrows $=$ Layers 1-4; arrowheads = cross-sections of vascular smooth muscle cells of Layer 2; white asterisk = a fibroblastic cell of Layer 1, and black asterisks = endothelial cells of Layer 4. (D) The longitudinal section cuts the vessel wall in the vicinity of Layer 3, the subendothelial layer, which primarily comprises finely dispersed smooth muscle cells and bundles of fibrils. White asterisks = dispersed smooth muscle cells of Layer 3; arrows = Layers 1, 2, and 4, respectively; arrowheads = cross-sections of vascular smooth muscle cells of Layer 2; black asterisk = endothelial cell in Layer 4. No sections are stained. Pseudocolor is caused by autofluorescence [17].

labeled the intravascular p-microcells intensively, which indicates a high concentration of hyaluronan receptors [21] and, consequently, depicts a large level of hyaluronic acid [22]. The blood vessel and lymphatic endothelial cell markers revealed that node vessels do not belong to blood or lymph vasculatures. RNA analysis showed that node has a very different gene transcript expression and representative gene distribution in comparison to the lymph node, bone marrow, and blood vessel. This implies that the node is different from the other three samples in terms of genetic nature [17].

To define the nature of cells that compose the bone marrow node, we used multiple antibodies. Fig. 5 shows the interplay of the node immunostained by the anti-fibronectin antibody. We defined three indicative areas in Fig. 5A, as areas 1,2, and 3 , and represented them magnified ten times in Fig. 5B-D, along with the control images, Fig. 5C-G. The cross-section of the sub-vessels shows fibers of Layer 1 and the smooth muscles of Layer 2 veining around the vessel (Fig. 5B-F). Fibronectin is closely associated with vascular smooth muscle cells [23]. A large density of p-microcells is clearly visible around sub-vessels in the magnified images.

\section{Nucleus-like structures and p-microcells of bone marrow node}

Dr. Bong-Han Kim used specific stains to visualize cell nuclei [15] and called the structures labeled by these stains nucleus-like structures. While describing morphological dynamics of primo nodes, he observed the assembling of relatively large nucleus-like structures from smaller components [13]. The dynamics of nucleus-like forms in primo node were reproduced in our time using DNA-stained DAPI [24]. The cross-section of the bone marrow node at high magnification typically shows nucleus-like structures alongside the dense population of p-microcells. Fig. 6 depicts two relatively large nucleus-like structures (asterisks) connected with p-microcells by the way of numerous cytoplasmic extensions. The large nucleus-like structure (left asterisk) stretched out a broad cytoplasmic extension to the 
nearest p-microcells, due to which it looks as if the nucleuslike structure draws in p-microcells. Smaller nucleus-like structures are also shown in the slide (white arrowheads).

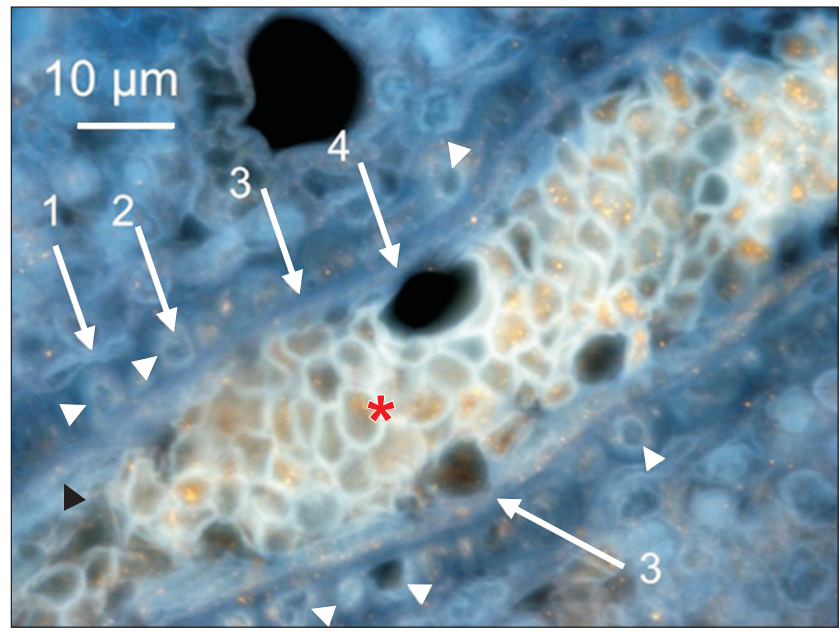

Fig. 4. Fluorescence images of cells within the longitudinal cross-sections of the primo node are immunostained with the anti-LYVE-1 antibody. The longitudinal section cuts the middle portion of the vessel. Red asterisk $=$ the p-microcells stained by the anti-LYVE-1 antibody; White arrows = Layers 1-4; white arrowheads = cross-sections of vascular smooth muscle cells of Layer 2; Black arrowhead $=$ endothelial cells of the Layer 4; Open triangle $=$ fibroblastic cell of Layer 1. Layer 4 of endothelial cells is not stained by an anti-LYVE-1 antibody.
P-microcells in this slide are of 2-3 microns in diameter and variable shape of nucleosome.

P-microcells inside the bone marrow primo node interact with different stem cell markers as they develop (Fig. 7). The anti-actin antibody stains all the microcells of about 2 microns in diameter. Anti-Nanog immunostains nucleus-like structures, the smallest p-microcells, as well as p-microcells of

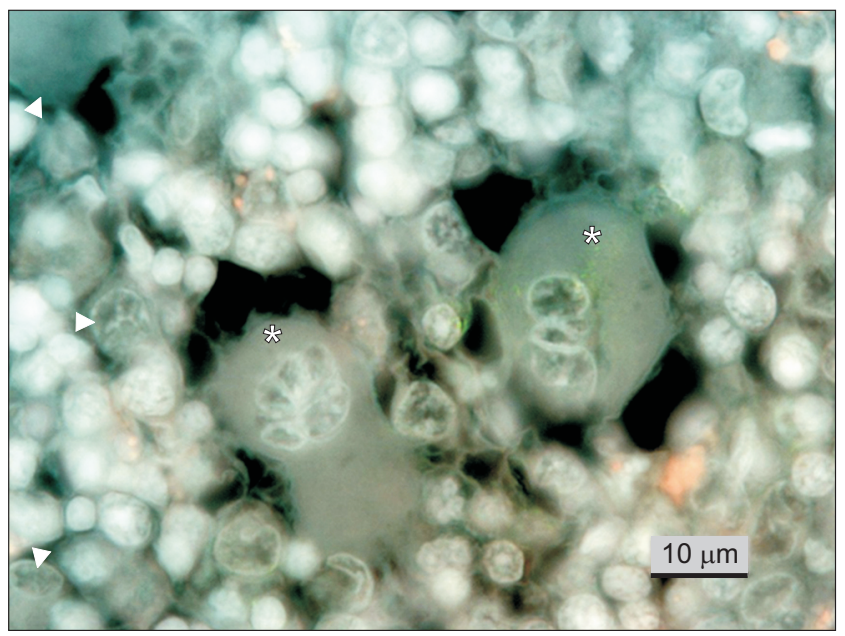

Fig. 6. Nucleus-like structures in the cross-section of bone marrow node. The nucleus-like structures (white arrowheads and white asterisks) of various sizes are surrounded by p-microcells.
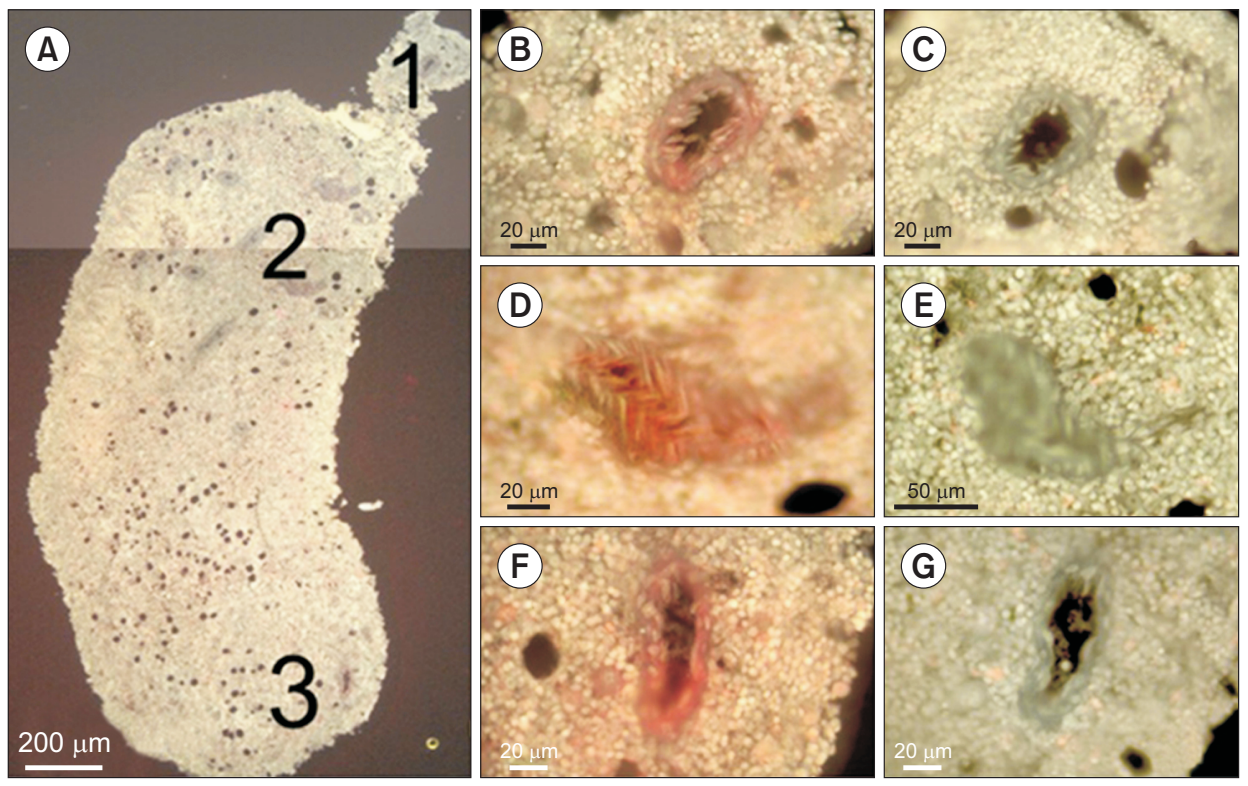

Fig. 5. Fluorescence images of cells in the cross-sections of primo node immunostained with the anti-fibronectin. (A) The image of the cross-sections of the primo node. Ten times enlarged areas 1, 2, and 3 stained with an anti-fibronectin antibody are revealed in panels ( $B, D$, and F), respectively, while panels (C, E, and $G$ ) show controls (no antibody) for the same areas. (B) Deposition of antibody on the transversal section of the primo node vessel in area 1 shows fibers in the external layer of the vessel (color reddish). (C) The sample is shown with no antibody (control). (D) The longitudinal section of the vessel in area 2. (E) Control (no antibody). (F) The longitudinal section traverses the middle part of the vessel in area 3, revealing immunostaining of external fibers around the vessel. (G) Control (no antibody). 

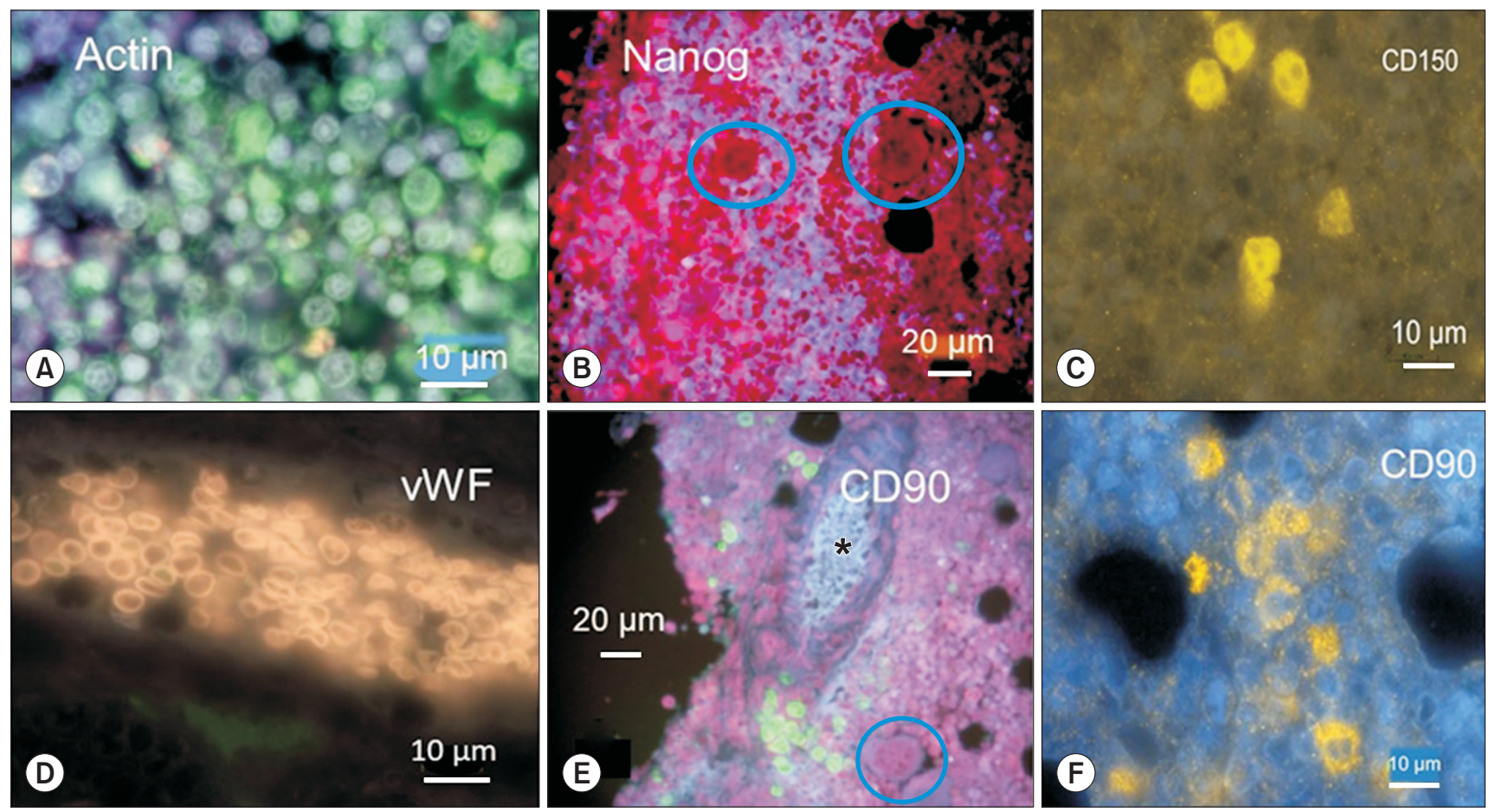

Fig. 7. P-microcells in bone marrow primo node stained by stem cell markers. (A) Actin, there are no filament or motherdaughter formations. (B) Nanog, blue circles = nucleus-like structures. (C) Bone marrow node labeled by CD150. (D) VWF, Von Willebrand Factor, p-microcells are inside primo node sub-vessel. (E) CD90, p-microcells stained green interact with the antibody. Nucleus-like structures (blue circle), scattered small p-microcells, and p-microcells in the sub-vessel (asterisk) do not interact with CD-90. (F) Bone marrow node labeled by CD90.

larger sizes. Anti-Von Willebrand Factor stains p-microcells inside sub-vessels. Anti-CD90 stains relatively larger pmicrocells and does not stain small p-microcells, p-microcells inside sub-vessels, or nucleus-like structures. Magnified images of p-microcells stained by CD90 and CD150 show two nuclei (Fig. 7C and F). There are no visible p-microcell filaments and mother-daughter cells. The Nanog frame (Fig. 7B) is composed of small and large nucleus-like structures.

\section{Organ surface primo node, vessels, and p-microcells}

The node is heterogeneous in structure, comprising twisted sub-vessel bundles that fill the volume of an almost entire node. Vascular bundles interlace in such a close-fitting structure that the node maintains its physical integrity even without the external capsule that was lost during sample preparation (Fig. 8A, a). The bundle of primo vessels $\left(\mathrm{B}_{\|}\right)$ on the right side of the section runs along the whole node body. The diameter of p-vessels in this bundle is $\sim 3 \mu \mathrm{m}$. The bundles $\left(\mathrm{B}_{\|}\right)$economically pack the node volume through serpentine-like structures. The image of the node is consistent with that shown in [20].

The magnified part of the primo node (Fig. 8A, b), similarly, demonstrates that the internal pattern of sub-vessels sustains the bundle-like design.

Fig. $8 \mathrm{~B}$ depicts the magnified area shown in the dotted rectangle of Fig. 8A, a. The diameter of the vessel is about 40 $\mu \mathrm{m}$. The lower-left portion of the vessel exposes the lumen (L) (Fig. 8B, a). The outer layer of the lumen shows nuclei of the fibroblasts of the longitudinal fiber (empty white arrowheads). The prominent traces of smooth muscle cells and fibers are further visible along the vessel length that looks like parallel streaks labeled with black arrowheads. These muscle cells and fibers have the appearance of coils around the sub-vessel. The nuclei of the dispersed longitudinal muscle-like cells are very noticeable (blue arrow). The characteristic rod-like structure (labeled by a green arrow) represents the nucleus of the endothelial cell. The layer of endothelial cells comprises the internal wall of the sub-vessel, whereas white arrowheads label a few p-microcells attached to the internal surface of the lumen.

The optical section of the sub-vessel positioned about one micron below the surface in Fig. 8B, a is shown in panel Fig. $8 \mathrm{~B}, \mathrm{~b}$. This section provides a better view of the nuclei of the endothelial and dispersed longitudinal muscle-like cells (green and blue arrows, respectively). The section also displays a few nuclei of the fibroblasts of the longitudinal fiber (empty white arrowheads) and many nuclei of the transversal 

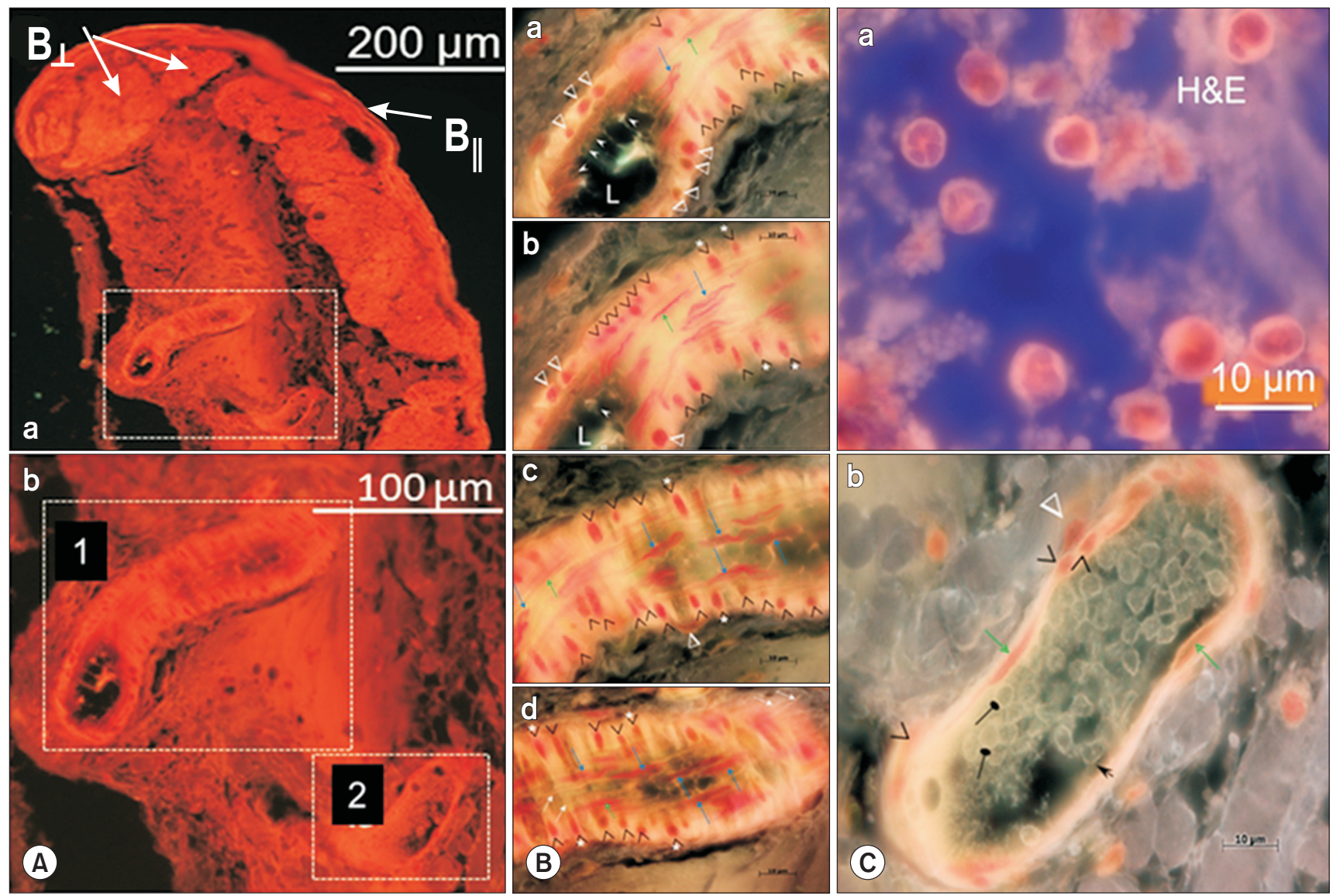

Fig. 8. Longitudinal sections of the primo node from the surface of a small intestine. (A) Primo node is a complex anastomosis of vessels. (a) $B_{\|}$is the longitudinal bundle of primo-capillaries; $B_{\perp}$ is the cross-sections of transversal vascular bundles. Two vessel sinuses surrounded by fibrous tissue are shown in the lower portion of the slide (dotted rectangle). (b) Magnified view of the rectangle in (A). Rectangle $1=$ top broaden sub-vessel, $2=$ lower sub-vessel. (B) Optical sections of the primo sub-vessel inside a primo node shown in (A) b; 1. (a) The optical section of the sub-vessel is about one micron below the cutting plane of the vessel and perpendicular to the microscope objective axis. (b) The optical section of the sub-vessel is a few microns below the surface in a. (c) The optical section of the sub-vessel is a few microns below the surface in b. (d) The optical section of the sub-vessel is about a few microns below the surface in c. White empty arrowheads = fibroblastic cells; white arrowheads $=p$-microcells; $\mathrm{L}=$ lumen; black arrowheads = circular vascular smooth muscle cells; black arrowheads with an asterisk = vascular smooth muscle cells that belong to the same coil; blue arrow = dispersed smooth muscle cells and collagen fibers; green arrow $=$ endothelial cells, dotted arrows $=$ thin $\sim 1 \mu \mathrm{m}$ fibers going along the length of the primo sub-vessel. (C) an H\&Estained p-microcells in the organ surface primo node in the histological slide. P-microcells are about 3-4 micrometers in diameter. (b) The dark field image of p-microcells in the sub-vessel of the organ surface primo node. Various arrows inside the vessels indicate p-microcells of different shapes. Black empty arrowheads = the nucleus of the fibroblasts of the longitudinal fiber; black empty arrowheads = nuclei of the fibroblasts of the fine transversal fiber; green arrow = nuclei of endothelial cells.

muscle cells (black arrowheads). The next optical section is again one micron closer to the external envelope (Fig. 8B, c). The images of the nuclei of endothelial and muscle-like cells become more notable than those in the former optical section. Finally, in the next optical section (Fig. 8B, d), which is very close to the internal part of the vessel membrane, the images of the nuclei of endothelial and longitudinal muscle-like cells become the most dominant features of the section. Fig. 8C shows p-microcells of organ surface primo nodes. P-microcells scattered in the slide have a multinuclear nucleosome and do not appear as a product of chromosome division (Fig. 8C, a). P-microcells inside the sub-vessels are depicted in Fig. 8C, b.

Immunohistochemistry (Table 1) of bone marrow primo nodes showed that it contains mesenchymal, hematopoietic, and endothelial stem cells. In addition to the stem cells, the node comprises many p-microcells scattered in the node cross-sections and inside vessels. Small p-microcells are accompanied by nucleus-like structures of about $25 \mu \mathrm{m}$ in diameter. The small p-microcells are clearly labeled and visible inside the large cells.

According to Kim, the primo vascular system is composed of nodes and vessels. Each primo vessel joins primo nodes together, and each primo node is linked with primo vessels 
Table 1. Distribution of cell markers in the bone marrow and sub-vessels

\begin{tabular}{|c|c|c|c|c|c|c|c|}
\hline Antibody & $\begin{array}{l}\text { Subvessel } \\
\text { layer } 1^{1}\end{array}$ & $\begin{array}{l}\text { Subvessel } \\
\text { layer } 2^{2}\end{array}$ & $\begin{array}{l}\text { Subvessel } \\
\text { layer } 3^{3}\end{array}$ & $\begin{array}{l}\text { Subvessel } \\
\text { layer } 4^{4}\end{array}$ & $\begin{array}{l}\text { P-microcells } \\
\text { in subvessel }\end{array}$ & $\begin{array}{l}\text { Nucleus-like } \\
\text { structure }^{6}\end{array}$ & $\begin{array}{c}\text { Scattered } \\
\text { p-microcells }\end{array}$ \\
\hline$\alpha$-Actin & - & + & - & - & - & - & - \\
\hline Actin & - & + & + & + & + & + & + \\
\hline CD146 & - & + & - & +- & + & -+ & - \\
\hline CD90 & - & -+ & - & - & + & - & + \\
\hline CD133 & - & - & - & - & + & + & + \\
\hline CD150 & - & + & - & +- & + & - & + \\
\hline Collagen-1 & + & - & - & +- & + & - & - \\
\hline Fibronectin & + & + & - & - & - & + & - \\
\hline LYVE-1 & - & - & - & - & + & - & - \\
\hline Nanog & + & + & - & - & + & - & + \\
\hline OCT4 & - & - & - & - & + & + & + \\
\hline RECA-1 & - & - & - & - & - & - & - \\
\hline REXO1 & - & - & - & - & - & - & + \\
\hline SOX2 & -+ & + & - & +- & - & +- & + \\
\hline SSEA-1 & - & - & - & - & - & - & + \\
\hline vWF & - & + & - & - & + & - & + \\
\hline
\end{tabular}

${ }^{1}$ The outermost layer of the vessel mainly consists of fibroblasts and fibrocytes, collagen, and fibrinogen fibers organized in longitudinal bundles. ${ }^{2}$ One of the middle layers is comprised of a three-dimensional network of transversally positioned smooth muscle cells. ${ }^{3}$ Layer is composed of thinly dispersed longitudinally oriented muscle-like cells. ${ }^{4}$ Innermost layer is comprised of endothelial cells. ${ }^{5}$ Cells inside sub-vessels. ${ }^{6}$ LNucleus-like structures. ${ }^{7}$ Cells scattered in the bone marrow node cross-section. $+-=$ most cells positive; $-=$ negative; $+-=$ often positive; $-+-=$ rarely positive .
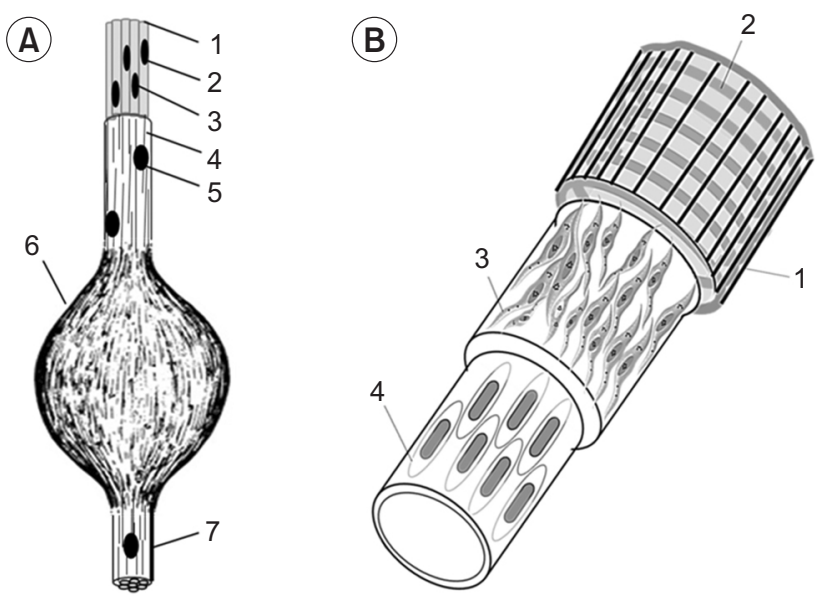

Fig. 9. Primo node, vessel, and sub-vessel. (A) Primo node, incoming and outcoming vessels; 1 = sub-vessel; 2 = cell nucleus of the external envelope; 3 = nucleus of endothelial cell; 4 = external jacket of the outcoming primo-vessel; $5=$ nucleus of jacket endothelial cell; $6=$ primo node; $7=$ out coming primo vessel [16]. (B) Subvessel. $1=$ the outermost layer of thin longitudinal fibers; 2 = vascular circle smooth muscle cells; 3 = longitudinal cells; and $4=$ endothelial cells; $\mathrm{L}=$ Lumen [17].

(Fig. 9). The primo vessel comprises a bundle of sub-vessels. The sub-vessel bundle of the incoming (afferent) vessel enters the node and branches into additional bundles (Fig. 9A). Based on the results of high-resolution microscopy and
RNA-Seq analysis of bone marrow nodes [17], we propose an idealized diagram of the node vessel structure (Fig. 9B). Layer 1, the outmost layer of the primo node vessel, primarily comprises fibroblastic cells directed longitudinally. The thickness of Layer 1 is approximately 3-5 $\mu \mathrm{m}$. Layer 2, one of the two middle layers of the node vessel, is apparently visible in almost all the sections and seems like a prominent structure; it mainly comprises vascular smooth muscle cells. These cells have an average diameter of $4.6 \mu \mathrm{m}$ and can extend up to $22-25 \mu \mathrm{m}$ in length. The cells are organized circumferentially around the vessel just underneath the outermost Layer 1, constituting a continuous helix. Layer 3 is another middle layer that primarily contains vascular smooth muscle cells. This layer comprises longitudinally oriented thinly dispersed smooth muscle cells and bundles of collagen fibrils. Layer 4 represents a continuous endothelium. On average, the endothelial cells are $5.7-\mu \mathrm{m}$ wide and $18.6-\mu \mathrm{m}$ long and are located parallel to the long axis of the vessel [17].

\section{DISCUSSION}

Earlier, we discovered that the vascularized node-like compartments in rat bone marrow meet the requirements of stem cell niche and are called hemmules [17]. A further structural and biochemical analysis of these nodes and comparison with known properties of the primo vascular system convinced us that hemmules are intra-organic, primo 
vascular nodes. Indeed, hemmules and primo nodes are small compartments of about $1 \mathrm{~mm}$ in size, covered with fine capsules. They have incoming and outgoing vessels. They are filled with vessels, extracellular matrix, and mesenchymal, hematopoietic, endothelial stem cells and contain biological entities that specifically correspond to primo nodesp-microcells and nucleus-like structures. The node vessels are stained by Trypan blue and have a high concentration of hyaluronic acid [13]. Four-layered structures of sub-vessels in the bone marrow node and organ surface node are almost identical. We found that nodes and vessels in the bone marrow do not interact with the lymphatic and blood vessel antibody markers, and they are different by genetic nature. The cells inside the bone marrow node interact with antibody markers of stem cells similar to those of the primo vascular system [25].

Although bone marrow and organ surface primo node are quite similar in anatomical structure and biochemical properties, there are considerably notable differences between the two. The extracellular matrix makes up the largest proportion of the entire volume of the bone marrow primo nodes, while the sub-vessels contribute the largest share of the volume of organ surface nodes. Additionally, p-microcells of different stages of development fill the entire volume of the bone marrow node, including the extracellular matrix and sub-vessels. In contrast, p-microcells are rarely found in the extracellular matrix, but they densely occupy the subvessels of organ surface nodes. We suggest that bone marrow nodes can produce a large number of new cells but slowly as compared to the organ surface nodes. The organ surface nodes are capable of yielding a small number of new cells at a fast pace and increasing their production in critical situations, specifically pertaining to the damage of bone marrow [26,27].

Bong-Han Kim has paid much attention to smooth muscle cells in primo vessels. While describing the histological structure of organ surface node, he revealed the abundance of smooth muscle cells, which he divided into outer circular layer and inner-longitudinal layer [15]. Those layers of smooth muscle cells have been reported here as Layers 2 and 3 of the bone marrow sub-vessel. The circular and latitudinal muscles are critical for the movement of primo fluid along the p-vessels. The p-fluid movement is carried out similar to that with food in the small intestine [28]. Peristalsis incorporates a wave that causes circular muscle contraction propagating downward. Both circular and longitudinal muscle layers participate in the circular muscle relaxations and the longitudinal muscle contractions. The overall speed of the passage of food in the small intestine is $1 \mathrm{~cm} / \mathrm{min}$ or $\sim 0.2$ $\mathrm{mm} / \mathrm{second}$. This value of $0.1-0.6 \mathrm{~mm} / \mathrm{s}$ is very well consistent with the movement of the fluid filled in the primo vessel, as measured by Bong-Han Kim with $\mathrm{P}^{32}$ [29]. The primo fluid speed was remeasured by the injection of Alcian blue dye into the organ-surface primo node of rabbits. The speed was measured as $0.3 \pm 0.1 \mathrm{~mm} / \mathrm{s}$, and the flow distance was up to $12 \mathrm{c}$ [30]. The authors concluded that fluid flow was due to the vessel peristaltic movement. Owing to the movement at such a high speed, p-microcells and stem cells can reach any point of the human body within an hour.

Bong-Han Kim presented two different models of cell regeneration. In the initial model, the replacement of dying cells occurred without cell division and DNA replication. The DNA of a dying cell is recycled and utilized for the creation of a new cell. The cell prepared to die packed its chromosomes in p-microcells with one chromosome in each p-microcell. Then, the cell expelled the cluster of $\mathrm{p}$-microcells into a primo vessel connected to the primo node, where p-microcells were combined into nucleus-like structures bearing the entire cell genome covered with a membranous layer. The nucleuslike structure matured when the internal p-microcells merged and, as a result, created a stem cell that was capable of replacing the dead cell [13]. In one regeneration cycle, one dying cell is replaced by a single new cell. This chromosomerecycling model rules out the probability of cell division or DNA synthesis and, therefore, confirms that it does not generate errors (mutations).

In the second model, the nucleic materials were also packed into p-microcells so that each chromosome is covered by a thin membrane and also includes a small amount of cytoplasm, mitochondria, and RNA. With a cell rupture, these p-microcells busted out of the cell, after which the fate of each p-microcells became independent. A p-microcell sent out a small filament of nucleosome outside the p-microcell membrane that grew into a daughter p-microcell. Similarly, he mother and daughter p-microcells formed numerous daughter p-microcells. When the bunch of p-microcells contained a full genome, it got covered with a membrane and developed a nucleus-like structure. Later, the borderline between $\mathrm{p}$-microcells became indistinct, and the nucleuslike structure turned into a stem cell that was ready for travel to make a new cell or replace the dead cell [13]. Theoretically, this regeneration model can produce as many new cells as the number of chromosomes in the dying cell. However, this model suggests DNA synthesis and, therefore, is associated with a possibility of errors. It is important that budding and protrusion of threadlike structure from $\mathrm{p}$-microcell is replicated in Dr. Soh's laboratory [31]. A similar finding was announced by Park et al. [25] in 2013.

We can develop a few criteria to differentiate between two different models of cell regeneration. For the chromosome synthesis (mother-daughter) model, we need to observe p-microcells with filaments and mother-daughter structures. We need to see large nucleus-like structures without any 
small nucleus-like structures having a membranous covering. For the chromosome recycling (DNA conservation) model, we should not see p-microcells with filaments and motherdaughter structures; however, we can see small and large nucleus-like structures covered with membranes.

Applying the above criteria to the bone marrow node, we have not seen obvious images of p-microcells with filaments or mother-daughter structures. However, we quite frequently observed the nucleus-like structures of different sizes (exemplified by Fig. 5 and Fig. 6B). These features meet the criteria of the chromosome recycling model of cell regeneration. Additionally, some p-microcells show two nuclei that seem like the product of merging of two small p-microcells and were likely produced by a DNA recycling mechanism (Fig. 6C, F). Thus, it is very likely that the regeneration of cells in the adult rat femur bone marrow intra-organic node aligns with the DNA recycling model. However, we believe that chromosome recycling and chromosome synthesis models are not mutually exclusive. In the present research, healthy adult animals required one dying cell to be replaced with a new one, which can be safely achieved by the chromosome recycling mechanism. When the system needs fast and multiple cell replacement, as in the case of wound healing, the chromosome synthesis model has an advantage over the chromosome-recycling model. To verify the chromosome-recycling model, we need to separate p-microcells and nucleus-like structures from the bone marrow node and determine whether or not a single p-microcell contains a single chromosome and the mature nucleus-like structure contains a whole genome. Additionally, we need to trace the path of p-microcells from the dying cell to the primo node and the route of the stem cell to the place of the expired cell.

Responding to the original question, "Why we all do not have a long and healthy life?", there are some cases of raised human longevity elucidated by exceptions to individual genetics [32], but the majority of us are not quite fortunate. We should not forget the exceptional property of the primo vascular system to regenerate our body without any mutation. We need to learn how to use and not abuse this system. It is easy to mistreat the PVS system with an improper diet and populated environment [33]. Supplementary research is required to determine ways to control PVS; however, ancient knowledge of Eastern medicine gives important lessons in this regard. Taking the idea that PVS is underpinning the acupuncture meridians, it can be presumed that the meridians form a three-dimensional vascular system that carries a special liquid Qi that contains p-microcells and stem cells [15]. Eastern medicine recognizes a phenomenon called Qi stagnation [34], which can be interpreted as difficulties in the movement of primo vascular fluid. There are medicinal herbs that may help with the "disorders in moving Qi" and can be found in many resources including two companion books $[35,36]$. Herbs such as Chai Hu (Bupleurum chinense $D C$.), Bai Shao (Paeonia lactiflora Pall.), Fu Ling (Poria Cocos (Schw) Wolf.), Bai Zhu (Atractylodes macrocephala Koidz.) are commonly used for moving Qi [37]. Additionally, acupoints ST36, LI4, and LR3 were found most commonly prescribed for 80 different diseases and conditions [38]. We hope in the future, PVS scientists (or PVS doctors) will be able to stimulate a system of primo nodes (including internal primo nodes) to direct stem cells to the required organs. A beginning has now been initiated in sending stem cells from the acupoint ST36 to the injured spinal cord of mice [39].

Overall, our results suggest that bone marrow nodes are intra-organic primo vascular nodes and offer methods and approaches to further evaluate these structures. Bone marrow nodes are easy to harvest and study ex-vivo in varying environmental conditions for assessing transition to human models, the regulation mechanism of cell regeneration, wound healing, and organism rejuvenation and longevity. If our conclusion is correct, studying these aspects, as well as other intra-organic nodes in animals and humans, may provide yet to be investigated methods of regenerative medicine and longevity.

\section{SUPPLEMENTARY MATERIAL}

Supplementary data to this article can be found online at https://doi.org/10.51507/j.jams.2022.15.1.12.

\section{FUNDING}

This work was supported by Grant \#2a from the Lake Erie College of Osteopathic Medicine, Erie, PA, USA.

\section{AUTHORS' CONTRIBUTIONS}

Conceptualization, V.V.; methodology, V.V. software, O.P.; formal analysis, V.V; data curation, O.P., L.G; writingoriginal draft, V.V.; writing_-review and editing, V.V.; project administration, V.V.

\section{CONFLICT OF INTEREST}

The authors declare no conflict of interest.

\section{ORCID}

Vitaly Vodyanoy, https://orcid.org/0000-0003-3092-6245 


\section{REFERENCES}

1. Levi CA, Ejere VC, Asogwa CN, Iweh P, Nwatu KU, Levi UE. Apoptosis: its physiological implication and therapeutic possibilities. IOSR J Pharm Biol Sci 2014;9(1):38-45.

2. Bianconi E, Piovesan A, Facchin F, Beraudi A, Casadei R, Frabetti F, et al. An estimation of the number of cells in the human body. Ann Hum Biol 2013;40:463-71. Erratum in: Ann Hum Biol 2013; 40:471.

3. Pray L. DNA replication and causes of mutation. Nat Educ 2008; 1:214.

4. Johnson RE, Washington MT, Prakash S, Prakash L. Fidelity of human DNA polymerase eta. J Biol Chem 2000;275:7447-50.

5. Sikora E, Bielak-Zmijewska A, Mosieniak G. Cellular senescence in ageing, age-related disease and longevity. Curr Vasc Pharmacol 2014;12:698-706.

6. Harley CB, Futcher AB, Greider CW. Telomeres shorten during ageing of human fibroblasts. Nature 1990;345:458-60.

7. Tzanetakou IP, Katsilambros NL, Benetos A, Mikhailidis DP, Perrea DN. "Is obesity linked to aging?": adipose tissue and the role of telomeres. Ageing Res Rev 2012;11:220-9.

8. Matthews C, Gorenne I, Scott S, Figg N, Kirkpatrick P, Ritchie A, et al. Vascular smooth muscle cells undergo telomere-based senescence in human atherosclerosis: effects of telomerase and oxidative stress. Circ Res 2006;99:156-64.

9. Sharpless NE, Schatten G. Stem cell aging. J Gerontol A Biol Sci Med Sci 2009;64:202-4.

10. Janzen V, Forkert R, Fleming HE, Saito Y, Waring MT, Dombkowski DM, et al. Stem-cell ageing modified by the cyclindependent kinase inhibitor p16INK4a. Nature 2006;443:421-6.

11. Pescatore LA, Gamarra LF, Liberman M. Multifaceted mechanisms of vascular calcification in aging. Arterioscler Thromb Vasc Biol 2019;39:1307-16.

12. Brownlee M. Advanced protein glycosylation in diabetes and aging. Annu Rev Med 1995;46:223-34.

13. Kim BH. Kyungrak System and Theory of Sanal. Pyongyang: Medical Science Press, 1965.

14. Soh KS, Kang KA, Ryu YH. 50 years of bong-han theory and 10 years of primo vascular system. Evid Based Complement Alternat Med 2013;2013:587827.

15. Vodyanoy V, Pustovyy O, Globa L, Sorokulova I. Primovascular system as presented by Bong Han Kim. Evid Based Complement Alternat Med 2015;2015:361974.

16. Vodyanoy V, Pustovyy O, Globa L, Sorokulova I. Evaluation of a new vasculature by high resolution light microscopy: primo vessel and node. ArXiv [Preprint]. 2016 Available from: https:// arxiv.org/abs/1608.04276.

17. Vodyanoy V, Pustovyy O, Globa L, Kulesza RJ Jr, Sorokulova I. Hemmule: a novel structure with the properties of the stem cell niche. Int J Mol Sci 2020;21:539.

18. Kang KA, Pustovyy O, Globa L, Sorokulova I, Vodyanoy V.
Sanal-Cell Cycle and Primo Vascular System: regeneration via sanals. Adv Exp Med Biol 2018;1072:413-8.

19. Vainrub A, Pustovyy O, Vodyanoy V. Resolution of $90 \mathrm{~nm}$ (lambda/5) in an optical transmission microscope with an annular condenser. Opt Lett 2006;31:2855-7.

20. Vodyanoy V. Characterization of primo nodes and vessels by high resolution light microscopy. In: Soh KS, Kang KA, Harrison DK, eds. The Primo Vascular System: Its Role in Cancer and Regeneration. New York: Springer, 2012:83-94.

21. Banerji S, Ni J, Wang SX, Clasper S, Su J, Tammi R, et al. LYVE1, a new homologue of the CD44 glycoprotein, is a lymphspecific receptor for hyaluronan. J Cell Biol 1999;144:789-801.

22. Kim BH. The Kyungrak system. J Jo Sun Med 1965;108:1-38.

23. Glukhova MA, Frid MG, Shekhonin BV, Vasilevskaya TD, Grunwald J, Saginati M, et al. Expression of extra domain A fibronectin sequence in vascular smooth muscle cells is phenotype dependent. J Cell Biol 1989;109:357-66.

24. Kim HG, Lee BC, Lee KB. Essential experimental methods for identifying Bonghan systems as a basis for Korean medicine: focusing on visual materials from original papers and modern outcomes. Evid Based Complement Alternat Med 2015;2015: 682735 .

25. Park ES, Lee JH, Kim WJ, Heo J, Shin DM, Leem CH. Expression of stem cell markers in primo vessel of rat. Evid Based Complement Alternat Med 2013;2013:438079.

26. Kim BH. Sanal and hematopoiesis. J Jo Sun Med 1965;108:1-6.

27. Shen Y, Lim CJ, Lee SY, Ryu PD. Acute anemia induces erythropoiesis in rat organ surface primo-vascular tissue. Adv Exp Med Biol 2020;1232:385-92.

28. Josephine N. Biology form 4 chapter 6 - nutrition part 2. Available at: https://www.slideshare.net/nirmalajosephine1/biology-form-4-chapter-6-nutrition-part-2 [Date accessed: June 20, 2021]

29. Kim BH. On the Kyungrak system. J Acad Med Sci DPR Korea 1963;90:1-35.

30. Sung B, Kim MS, Lee BC, Yoo JS, Lee SH, Kim YJ, et al. Measurement of flow speed in the channels of novel threadlike structures on the surfaces of mammalian organs. Naturwissenschaften 2008;95:117-24.

31. Baik KY, Ogay V, Jeoung SC, Soh KS. Visualization of Bonghan microcells by electron and atomic force microscopy. J Acupunct Meridian Stud 2009;2:124-9.

32. Schächter F. Causes, effects, and constraints in the genetics of human longevity. Am J Hum Genet 1998;62:1008-14.

33. Zhang GX, Jin L, Jin H, Zheng GS. Influence of dietary components and traditional Chinese medicine on hypertension: a potential role for gut microbiota. Evid Based Complement Alternat Med 2021;2021:5563073.

34. Zhai X, Feng XC, Liu JW, Gao K, Jia ZH, Zhao HH, et al. Neuro-endocrine-immune biological network construction of qi deficiency pattern and qi stagnation pattern in traditional 
Chinese medicine. J Biol Syst 2015;23:305-21.

35. Hsu H, Hsu C. Commonly Used Chinese Herb Formulas with Illustrations. Los Angeles: Oriental Healing Arts Institute, 1980. $671 \mathrm{p}$.

36. Hsu H, Van Benschoten MM. Index of Differentiations for Commonly Used Herb Formulations. Long Beach: Oriental Healing Arts Institute, 1984. 257 p.

37. Yeung WF, Chung KF, Ng KY, Yu YM, Zhang SP, Ng BF, et al. Prescription of Chinese herbal medicine in pattern-based traditional Chinese medicine treatment for depression: a syste- matic review. Evid Based Complement Alternat Med 2015;2015: 160189.

38. Lee YS, Ryu Y, Yoon DE, Kim CH, Hong G, Hwang YC, et al. Commonality and specificity of acupuncture point selections. Evid Based Complement Alternat Med 2020;2020:2948292.

39. Jung SJ, Kook MG, Kim S, Kang KS, Soh KS. Homing of the stem cells from the acupoint ST-36 to the site of a spinal cord injury: a preliminary study. J Acupunct Meridian Stud 2018;11: 133-6. 